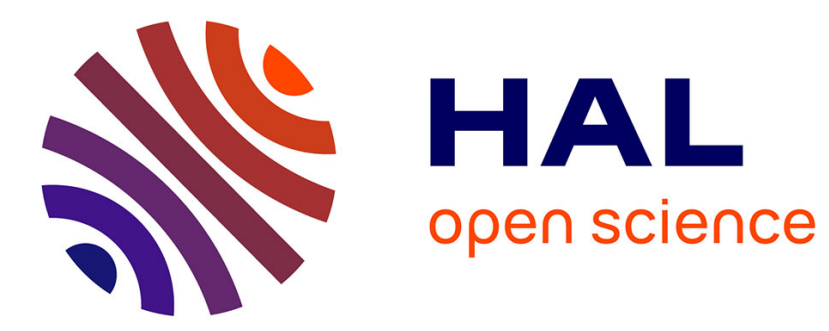

\title{
Non linéarité des fonctions booléennes données par des traces de polynômes de degré binaire 3
}

\author{
François Rodier, Eric Férard
}

\section{To cite this version:}

François Rodier, Eric Férard. Non linéarité des fonctions booléennes données par des traces de polynômes de degré binaire 3. 2007. hal-00151591

\section{HAL Id: hal-00151591 \\ https://hal.science/hal-00151591}

Preprint submitted on 4 Jun 2007

HAL is a multi-disciplinary open access archive for the deposit and dissemination of scientific research documents, whether they are published or not. The documents may come from teaching and research institutions in France or abroad, or from public or private research centers.
L'archive ouverte pluridisciplinaire HAL, est destinée au dépôt et à la diffusion de documents scientifiques de niveau recherche, publiés ou non, émanant des établissements d'enseignement et de recherche français ou étrangers, des laboratoires publics ou privés. 


\title{
Non linéarité des fonctions booléennes données par des traces de polynômes de degré binaire 3
}

\author{
Eric Férard, François Rodier ${ }^{\dagger}$
}

\begin{abstract}
Résumé
Nous étudions la non linéarité des fonctions définies sur $\mathbf{F}_{2^{m}}$ où $m$ est un entier impair, associées aux polynômes de degré 7 ou à des polynômes plus généraux.
\end{abstract}

Keywords : fonction booléenne, non linéarité, indice de somme des carrés, courbe supersingulière de genre 2.

\section{Introduction}

La non-linéarité d'une fonction booléenne $f: \mathbf{F}_{2}^{m} \longrightarrow \mathbf{F}_{2}$ est la distance de $f$ à l'ensemble des fonctions affines à $m$ variables (voir les $\S$ 2.2). C'est un concept important.

Il intervient en cryptographie (cf. [3, 5, 6, 8]) pour construire des cryptosystèmes performants (chiffrements symétriques), et dans la théorie de codage avec le vieux problème du rayon de recouvrement des codes de ReedMuller d'ordre 1.

La non-linéarité est inférieure à $2^{m-1}-2^{m / 2-1}$. Cette limite est atteinte par les fonctions courbes (cf. le livre de MacWillams et de Sloane [15]) qui existent seulement si le nombre de variables $m$ des fonctions booléennes est pair. Pour des raisons de sécurité en cryptographie, et aussi parce que les

\footnotetext{
${ }^{*}$ Université de Polynésie française, Tahiti; e-mail ferard@upf .pf

${ }^{\dagger}$ Institut de Mathématiques de Luminy - C.N.R.S. 163 avenue de Luminy, Case 907, Marseille Cedex 9, France; e-mail rodier@iml.univ-mrs.fr; Tel 0491269589 ;Fax 04 91269655
} 
fonctions booléennes doivent avoir d'autres propriétés telles que l'équilibre ou le degré algébrique élevé, il est important d'avoir la possibilité de choix parmi beaucoup de fonctions booléennes, non seulement des fonctions courbes, mais également des fonctions presque courbes dans le sens que leur non-linéarité est voisine de la non-linéarité des fonctions courbes.

Pour $m$ impair, il serait particulièrement intéressant de trouver des fonctions avec une non-linéarité plus grande que celle de fonctions booléennes quadratiques (appelées presque optimales dans [2]). Ceci a été fait dans le travail de Patterson et de Wiedemann [16] et également de Langevin et Zanotti [11] et plus récemment par Kavut, Maitra et Yücel [12].

Soit $q=2^{m}$ et $k=\mathbf{F}_{2^{m}}$ assimilé comme espace vectoriel sur $\mathbf{F}_{2}$ à $\mathbf{F}_{2}^{m}$. Si $G$ est un polynôme sur $k$, cela nous permet de construire une fonction booléenne $\operatorname{Tr} G(x)$, où Tr est la trace de $\mathbf{F}_{2^{m}}$ sur $\mathbf{F}_{2}$, ou plutôt la fonction $\chi(G(x))$, avec des valeurs dans \pm 1 , où nous dénotons par $\chi_{0}$ le caractère non trivial unique de $\mathbf{F}_{2}$ dans les nombres complexes différents de zéro :

$$
\chi_{0}(0)=1 \quad, \quad \chi_{0}(1)=-1
$$

et nous notons $\chi=\chi_{0} \circ \mathrm{Tr}$.

Pour $m$ pair, on a cherché à trouver des fonctions courbes de cette forme. Pour mentionner seulement le cas des monômes, on peut considérer les cas connus (de Gold, de Dillon, des exposants de Niho) dans l'article de Leander [10]. Ce sont des fonctions $f: x \longrightarrow \chi\left(a x^{r}\right)$ où $r=3$ ou 5 (ou plus généralement $r=2^{i}+1$, où $i$ est un nombre entier) et $a \in k$ n'est pas de la forme $x^{r}$.

On aurait pu espérer que pour $r=7$, ou parmi les fonctions

$$
f: x \longrightarrow \chi(G(x))
$$

quand $G$ est un polynôme du degré 7 , il y a quelques fonctions qui sont presque courbes au sens précédent. Cela s'avère ne pas être le cas, mais nous prouverons que pour $m$ impair de telles fonctions ont les propriétés de nonlinéarité plutôt bonnes (cf. section 《). Nous employons pour cela des résultats récents de Maisner et de Nart au sujet des fonctions de zêta des courbes supersingulières de genre 2 que nous avons regroupés dans les sections 5, 6, 7 . 


\section{Préliminaires}

\subsection{Fonctions booléennes}

Soit $m$ un entier positif et $q=2^{m}$.

Définition 2.1 Une fonction booléenne à $m$ variables est une application de l'espace $V_{m}=\left(\mathbf{F}_{2}\right)^{m}$ dans $\mathbf{F}_{2}$.

Une fonction booléenne est linéaire si c'est une forme linéaire sur l'espace vectoriel $\left(\mathbf{F}_{2}\right)^{m}$. Elle est dite affine si elle est égale à une fonction linéaire à une constante près.

\subsection{Non-linéarité}

Définition 2.2 On appelle non-linéarité d'une fonction booléenne $f$ à $m$ variables et on la note $\mathrm{nl}(\mathrm{f})$ la distance qui la sépare de l'ensemble des fonctions affines à $m$ variables :

$$
n l(f)=\min _{h \text { affine }} d(f, h)
$$

où d est la distance de Hamming.

On peut prouver que la non-linéarité est égale à

$$
n l(f)=2^{m-1}-\frac{1}{2}\|\widehat{f}\|_{\infty}
$$

où

$$
\|\widehat{f}\|_{\infty}=\sup _{v \in V_{m}}\left|\sum_{x \in V_{m}} \chi_{0}(f(x)+v \cdot x)\right|
$$

et $v \cdot x$ denote le produit scalaire usuel de $V_{m}$. C'est le maximum de la transformée de Fourier de $\chi_{0}(f)$ (ou la transformée de Walsh de $f$ ) :

$$
\widehat{f}(v)=\sum_{x \in V_{m}} \chi_{0}(f(x)+v \cdot x) .
$$

On appellera $\|\widehat{f}\|_{\infty}$ l'amplitude spectrale de la fonction booléenne $f$. La formule d'inversion est donnée par

$$
\chi_{0}(f(x))=\frac{1}{q} \sum_{v \in V_{m}} \widehat{f}(v) \chi_{0}(v \cdot x)
$$


où l'on remarque que le dual de $V_{m}$ est isomorphe à $V_{m}$, avec la mesure $\frac{1}{q}$ sur chaque point. L'identité de Parseval peut s'écrire

$$
\|\widehat{f}\|_{2}^{2}=\frac{1}{q} \sum_{v \in V_{m}} \widehat{f}(v)^{2}=q
$$

et, si $f$ est une fonction booléenne sur $\mathbf{F}_{2}^{m}$ :

$$
\sqrt{q} \leq\|\widehat{f}\|_{\infty} \leq q .
$$

\subsection{L'indice de somme des carrés}

Soit $f$ une fonction booléenne sur $V_{m}$. Zhang et Zheng ont introduit l'indice de somme des carrés [24]:

$$
\sigma_{f}=\frac{1}{q} \sum_{x \in V_{m}} \widehat{f}(x)^{4}=\|\widehat{f}\|_{4}^{4}
$$

Nous remarquons que

$$
\|\widehat{f}\|_{2} \leq\|\widehat{f}\|_{4} \leq\|\widehat{f}\|_{\infty}
$$

La relation de cette fonction avec la non linéarité a été étudiée par A. Canteaut et al. [2]

\section{Les fonctions $f: x \longrightarrow \operatorname{Tr}(G(x))$ où $G$ est un polynôme}

\subsection{Divisibilité de $\|\widehat{f}\|_{\infty}$}

Soit $G(x)$ le polynôme $\sum_{i=0}^{s} a_{i} x^{i}$ à coefficients dans $\mathbf{F}_{q}$ et $f$ la fonction booléenne $\operatorname{Tr} \circ G$.

Définition 3.1 Le degré binaire de $G$ est la valeur maximum des $\sigma(i)$ pour $0 \leq i \leq s$, où $\sigma(i)$ est la somme des chiffres de $i$ écrit en chiffre binaire.

On a la proposition suivante, due à C. Moreno et O. Moreno [13], généralisant le théorème d'Ax.

Proposition 3.1 Soit $G$ un polynôme à coefficients dans $\mathbf{F}_{q}$, de degré binaire d. Alors $\|\widehat{f}\|_{\infty}$ est divisible par $2^{\left\lceil\frac{m}{d}\right\rceil}$. 


\subsection{Cas où $G$ est un polynôme de degré binaire 2}

Les $\|\widehat{f}\|_{\infty}$ sont multiples de $2^{\left\lceil\frac{m}{2}\right\rceil}$. Donc, si $m$ est pair $\|\widehat{f}\|_{\infty}$ est un multiple de $q^{1 / 2}$, et si $m$ est impair, de $\sqrt{2 q}$. En particulier, si $m$ est impair, l'amplitude spectrale est supérieure ou égale à $\sqrt{2 q}$ qui est égale à celle des fonctions booléennes quadratiques de rang maximal.

\section{Les fonctions $f: x \longrightarrow \operatorname{Tr}(G(x))$ où $G$ est un polynôme de degré binaire 3}

On va simplement étudier le cas où $G$ est un polynôme de degré binaire 2 auquel on a rajouté un monôme non nul de degré 7 , c'est-à-dire un polynôme de la forme

$$
G=a_{7} x^{7}+\sum_{0}^{s} b_{i} x^{2^{i}+1}
$$

où $a_{7} \neq 0$ un polynôme de degré 7 à coefficients dans $k$. Nous voudrions évaluer $\|\widehat{f}\|_{4}$ sur $\mathbf{F}_{2^{m}}$, pour $f(x)=\operatorname{Tr}(G(x))$ où Tr dénote la fonction trace de $\mathbf{F}_{q}$ vers $\mathbf{F}_{2}$ :

$$
\operatorname{Tr}(x)=\sum_{i=0}^{m-1} x^{2^{i}} .
$$

\subsection{Evaluation de $\|\widehat{f}\|_{4}^{4}$}

Proposition 4.1 La valeur de $\|\widehat{f}\|_{4}^{4}$ sur $\mathbf{F}_{2^{m}}$ quand $m$ est impair et $f(x)=$ $\chi(G(x))$ est telle que

$$
\left|\|\widehat{f}\|_{4}^{4}-3 q^{2}\right| \leq 185.2^{s-1} q^{3 / 2}
$$

Démonstration -

La démonstration sera donné dans la section 6 .

Remarque 4.1 Ce résultat est à comparer avec la proposition 5.6 de [1X] où on a montré que la distribution de $\|\widehat{f}\|_{4}^{4}$ pour toutes les fonction booléennes est concentrée autour de $3 q^{2}$.

\subsection{Bornes de $\|\widehat{f}\|_{\infty}$}

La démonstration de ces bornes seront données dans la section 0 . 


\subsubsection{Borne inférieure}

Proposition 4.2 Pour les fonctions $f: x \longrightarrow \chi(G(x))$ sur $\mathbf{F}_{2^{m}}$ où $G$ est un polynôme donné au début de la section $\mathbf{6}$ et $m$ est impair, on a, pour $m \leq 11+2 s$

$$
\sqrt{2 q} \leq\|\widehat{f}\|_{\infty}
$$

Pour $m \geq 15+2 s$, on a de plus

$$
\sqrt{2 q}+2^{\left\lceil\frac{m}{3}\right\rceil} \leq\|\widehat{f}\|_{\infty} .
$$

Remarque 4.2 Il est connu que pour $m$ impair et plus petit que 7 , on a $\sqrt{2 q} \leq\|\widehat{f}\|_{\infty}$ pour toutes les fonctions booléennes. [9]

\subsubsection{Borne supérieure}

Proposition 4.3 On a

$$
\|\widehat{f}\|_{\infty} \leq 6 \sqrt{q}
$$

\section{$5 \quad$ Etude de courbes hyperelliptiques}

Pour démontrer les résultats précédents, on va étudier des courbes liées au polynôme $G$.

On obtient d'abord l'expression simple de $\|\widehat{f}\|_{4}$ (cf. [17, 18]) :

$$
\|\widehat{f}\|_{4}^{4}=\sum_{x_{1}+x_{2}+x_{3}+x_{4}=0} \chi\left(f\left(x_{1}\right)+f\left(x_{2}\right)+f\left(x_{3}\right)+f\left(x_{4}\right)\right)=q^{2}+\sum_{\substack{\alpha \neq 0 \\ \alpha \in V_{m}}} X_{\alpha}
$$

avec

$$
X_{\alpha}=\left(\sum_{x \in k} \chi(G(x)+G(x+\alpha))\right)^{2} .
$$

On note maintenant $\alpha$ un élément de $k^{*}$. On peut vérifier que

$$
\begin{gathered}
G(x+\alpha)+G(x)=G(\alpha)+a_{7} \alpha^{6} x+a_{7} \alpha^{5} x^{2}+a_{7} \alpha^{4} x^{3}+a_{7} \alpha^{3} x^{4}+a_{7} \alpha^{2} x^{5}+ \\
a_{7} \alpha x^{6}+\sum_{0}^{s} b_{i}\left(\alpha x^{2^{i}}+\alpha^{2^{i}} x\right)
\end{gathered}
$$


Pour calculer $X_{\alpha}$, on peut remarquer que la courbe d'équation $y^{2}+y=$ $G(x+\alpha)+G(x)$ est isomorphe à

$$
\begin{aligned}
y^{2}+y & =G(\alpha)+ \\
+ & \left(a_{7} \alpha^{6}+a_{7}^{1 / 4} \alpha^{3 / 4}+a_{7}^{1 / 2} \alpha^{5 / 2}+\sum_{0}^{s}\left(b_{i} \alpha\right)^{2^{-i}}+\sum_{0}^{s} b_{i} \alpha^{2^{i}}\right) x+ \\
& +\left(a_{7} \alpha^{4}+a_{7}^{1 / 2} \alpha^{1 / 2}\right) x^{3}+a_{7} \alpha^{2} x^{5}
\end{aligned}
$$

qui est une équation de la courbe $C_{1}$ de genre 2 pour $\alpha \neq 0$.

On a

$$
X_{\alpha}=\left(\# C_{1}-q-1\right)^{2} .
$$

\subsection{La théorie de van der Geer et van der Vlugt}

Soit $C_{1}$ la courbe d'équation affine :

$$
C_{1}: y^{2}+y=a x^{5}+b x^{3}+c x+d
$$

avec $a \neq 0$. Soit $R$ le polynôme linéaire $a x^{4}+b x^{2}+c^{2} x$. L'application

$$
\begin{aligned}
Q: k & \longrightarrow \mathbf{F}_{2} \\
x & \longmapsto \operatorname{Tr}(x R(x))
\end{aligned}
$$

est la forme quadratique associé à la forme symplectique

$$
\begin{aligned}
& k \times k \quad \mathbf{F}_{2} \\
& (x, y) \longmapsto<x, y>=\operatorname{Tr}(x R(y)+y R(x)) .
\end{aligned}
$$

Le nombre de zéros de $Q$ détermine le nombre de points de $C_{1}$ :

$$
\# C_{1}(k)=1+2 \# Q^{-1}(0)
$$

Le radical $W$ de la forme symplectique $<,>$ concide avec l'ensemble des zéros dans $k$ du polynôme $\mathbf{F}_{2}$-linéaire et séparable

$$
E_{a, b}=a^{4} x^{16}+b^{4} x^{8}+b^{2} x^{2}+a x .
$$

On a : $0 \leq w=\operatorname{dim}_{\mathbf{F}_{2}} W \leq 4$ et $w \equiv m$ (mod. 2$)$. La codimension du noyau $V$ de $Q$ dans $W$ est égale à 0 ou 1 . De plus, le polynôme $E_{a, b}$ se factorise dans $k[x]([22]$, Theorem 3.4) :

$$
E_{a, b}(x)=x P(x)\left(1+x^{5} P(x)\right)
$$

avec $P(x)=a^{2} x^{5}+b^{2} x+a$. 
Théorème 5.1 (van der Geer - van der Vlugt [29])

Si $V \subset W$, alors $\# C_{1}(k)=1+q$.

Si $V=W$, alors $\# C_{1}(k)=1+q \pm \sqrt{2^{w} q}$.

\subsection{Les travaux de Maisner et Nart}

Supposons que $a=b$ et que le polynôme $P$ ait au moins une racine $z$. Alors, comme $m$ est impair, il existe un unique $\ell \in k$ tel que $\ell^{3}=1+z^{-4}$.

Proposition 5.1 Si $\operatorname{Tr} \ell=0$ alors le polynôme $P$ a exactement trois racines dans $k$ et on a $w=3$. Si $\operatorname{Tr} \ell \neq 0$ alors le polynôme $P$ n'a qu'une racine dans $k$, la composante restante est irréductible et on a $w=1$.

Démonstration -

Voir Maisner et Nart [14] propositions 2.3 et 2.6.

\subsection{Réduction de la courbe $y^{2}+y=G(x+\alpha)+G(x)$}

Soit $\lambda=\alpha+a_{7}^{-1 / 4} \alpha^{-3 / 4}$.

\subsubsection{Cas où $\lambda=0$}

Alors on a $\alpha^{7}=a_{7}^{-1}$, donc l'équation de la courbe devient

$$
\begin{aligned}
y^{2}+y= & G(\alpha)+\left(a_{7} \alpha^{6}+a_{7}^{1 / 4} \alpha^{3 / 4}+a_{7}^{1 / 2} \alpha^{5 / 2}+\sum_{0}^{s} b_{i}\left(\alpha^{2^{-i}}+\alpha^{2^{i}}\right)\right) x+ \\
& \quad+a_{7} \alpha^{2} x^{5} \\
= & d+c x+a x^{5}
\end{aligned}
$$

pour $a=\alpha^{-5}$.

Le polynôme $P$ s'écrit $P(x)=a^{2} x^{5}+a$. Si $m$ est impair il a une unique racine $z=a^{-1 / 5}=\alpha$. D'après Maisner et Nart ([14], Propositions 2.5 et 2.3) on est dans le cas où $w=1$ donc $W=\{0, z\}$. Soit $c$ le coefficient de $x$. On a

$$
\begin{aligned}
\operatorname{Tr}(c z) & =\operatorname{Tr}\left(\left(1 / \alpha+a_{7}^{1 / 4} \alpha^{3 / 4}+a_{7}^{1 / 2} \alpha^{5 / 2}+\sum_{0}^{s}\left(b_{i} \alpha\right)^{2^{-i}}+\sum_{0}^{s} b_{i} \alpha^{2^{i}}\right) \alpha\right) \\
& =\operatorname{Tr}\left(1+\sum_{0}^{s} b_{i}^{2^{-i}} \alpha^{\frac{2^{i}+1}{2^{i}}}+\sum_{0}^{s} b_{i} \alpha^{1+2^{i}}\right) \\
& =\operatorname{Tr} 1=1
\end{aligned}
$$


On vérifie alors que

$$
Q(z)=\operatorname{Tr}\left(a z^{5}+c z\right)=\operatorname{Tr}(1+c z)=0
$$

D'où $V=W$ et donc $X_{\alpha}=2 q$ par le théorème 5.1.

\subsubsection{Cas où $\lambda \neq 0$}

Cette courbe est isomorphe à

$$
y^{2}+y=a x^{5}+a x^{3}+c x+d
$$

avec

$$
a=\lambda^{5} a_{7} \alpha^{2}=\lambda^{3}\left(a_{7} \alpha^{4}+a_{7}^{1 / 2} \alpha^{1 / 2}\right)
$$

et $\lambda=\alpha+a_{7}^{-1 / 4} \alpha^{-3 / 4}$. On a

$$
a=1+a_{7}^{-1 / 4} \alpha^{-7 / 4}+a_{7}^{3 / 4} \alpha^{21 / 4}+a_{7} \alpha^{7}
$$

et

$$
c=1+\left(\sum_{0}^{s}\left(b_{i} \alpha\right)^{2^{-i}}+\sum_{0}^{s} b_{i} \alpha^{2^{i}}\right) \lambda+a_{7}^{1 / 2} \alpha^{7 / 2}+a_{7}^{3 / 4} \alpha^{21 / 4}+a_{7} \alpha^{7} .
$$

\subsection{Valeurs de $X_{\alpha}$}

Proposition 5.2 Supposons que $m$ soit impair. Alors $X_{\alpha}=0,2 q$ ou $8 q$. Soit $\ell=a_{7}^{-1 / 3} \alpha^{-7 / 3}$. Alors

$$
\begin{array}{lc}
X_{\alpha}=8 q \quad & \text { si et seulement si } \\
& \operatorname{Tr} \ell=0 \quad, \quad \ell=v+v^{4} \quad, \\
& \operatorname{Tr}\left(\eta v^{3}\right)=1 \quad, \quad \operatorname{Tr}\left(\eta\left(v+v^{2}\right)\right)=1 ; \\
& \text { avec } \eta=1+\sum_{0}^{s}\left(b_{i} \alpha^{1+2^{i}}\right)^{2^{-i}}+\sum_{0}^{s} b_{i} \alpha^{1+2^{i}}+ \\
& +a_{7}{ }^{1 / 2} \alpha^{7 / 2}+a_{7}^{1 / 4} \alpha^{7 / 4} ; \\
X_{\alpha}=2 q \quad \text { si et seulement si } \operatorname{Tr} \ell=1 & ; \\
X_{\alpha}=0 \quad \text { dans les cas restant. }
\end{array}
$$


Démonstration -

Si $\lambda=0$, alors $\ell=1$ d'où $\operatorname{Tr} \ell=1$. On a bien $X_{\alpha}=2 q$ d'après 5.3.1.

Si $\lambda \neq 0$, on étudie le polynôme $P=a^{2} x^{5}+a^{2} x+a$. Remarquons que $z=\lambda^{-1} \alpha$ est racine de $P$. Donc

$$
\begin{aligned}
P & =(x+z)\left(a^{2} x^{4}+a^{2} x^{3} z+a^{2} x^{2} z^{2}+a^{2} x z^{3}+a^{2} z^{4}+a^{2}\right) \\
& =a^{2} z^{-4}(x+z)\left(x^{4} z^{-4}+x^{3} z^{-3}+x^{2} z^{-2}+x z^{-1}+z^{-4}+1\right) .
\end{aligned}
$$

La décomposition de $P$ en composante irréductibles dépend de $e=1+z^{-4}$. On a

$e=1+z^{-4}=1+\lambda^{4} \alpha^{-4}=1+\left(\alpha^{4}+a_{7}^{-1} \alpha^{-3}\right) \alpha^{-4}=1+\left(1+a_{7}^{-1} \alpha^{-7}\right)=a_{7}^{-1} \alpha^{-7}$.

Comme $m$ est impair, on a $k^{3}=k$. Soit $\ell=e^{1 / 3}$. Alors, d'après la proposition 5.1, on a

$$
\left\{\begin{array}{lll}
w=1 & \text { si } & \operatorname{Tr} \ell=1 \\
w=3 & \text { si } & \operatorname{Tr} \ell=0
\end{array}\right.
$$

D'après le théorème 5.1, on a

dans le premier cas, $X_{\alpha}=0$ ou $2 q$.

dans le deuxième cas, $X_{\alpha}=0 \quad$ ou $\quad 8 q$.

Premier cas, $\operatorname{Tr} \ell=1$. On a $W=\{0, z\}$ et

$$
Q(z)=\operatorname{Tr}\left(a z^{5}+a z^{3}+c z\right)=\operatorname{Tr}(a z+c z+1)
$$

car $\operatorname{Tr}\left(a z^{3}\right)=0$. Pour que $X_{\alpha}=0$ il faut et il suffit que $\operatorname{Tr}(a+c) z=0$. Des équations (2) et (3) on déduit

$$
\begin{aligned}
(a+c) z & =1+a_{7}^{1 / 4} \alpha^{7 / 4}+a_{7}^{1 / 2} \alpha^{7 / 2}+\left(\sum_{0}^{s} b_{i} \alpha^{2^{-i}}+\sum_{0}^{s} b_{i} \alpha^{2^{i}}\right) \alpha \\
& =1+a_{7}^{1 / 4} \alpha^{7 / 4}+a_{7}^{1 / 2} \alpha^{7 / 2}+\left(\sum_{0}^{s}\left(b_{i} \alpha^{1+2^{i}}\right)^{2^{-i}}+\sum_{0}^{s} b_{i} \alpha^{1+2^{i}}\right)
\end{aligned}
$$

Donc $\operatorname{Tr}((a+c) z)=\operatorname{Tr} 1=1$ et $X_{\alpha}=2 q$. 
Deuxième cas, $\operatorname{Tr} \ell=0$. On a $W=<z, z_{1}, z_{2}>$.

Pour que $X_{\alpha}=0$ il faut et il suffit que $\operatorname{Tr}(a+c) z_{i}=0$ pour l'un des $i=1,2$ ou que $\operatorname{Tr}(a+c) z=0$.

Les nombres $z_{i}$ sont racines de $x^{4} z^{-4}+x^{3} z^{-3}+x^{2} z^{-2}+x z^{-1}+z^{-4}+1=0$. On a $e=1+z^{-4}=\ell^{3}$ et $\ell=u+u^{2}$. D'où, d'après Maisner et Nart 14 (démonstration du lemme 2.4) :

$$
\begin{aligned}
& x^{4} z^{-4}+x^{3} z^{-3}+x^{2} z^{-2}+x z^{-1}+z^{-4}+1= \\
& \left(x^{2} z^{-2}+u x z^{-1}+(1+u)^{3}\right)\left(x^{2} z^{-2}+(u+1) x z^{-1}+u^{3}\right)
\end{aligned}
$$

On peut supposer $\operatorname{Tr} u=0$ (car $\operatorname{Tr} 1=1$, donc $u$ ou $1+u$ a une trace nulle). Soit donc $u=v+v^{2}$. On a par conséquent $\ell=v+v^{4}$. Alors le polynôme $x^{2} z^{-2}+(u+1) x z^{-1}+u^{3}$ est réductible : ses racines sont : $z(v(1+u)+1)=$ $z\left(v\left(1+v+v^{2}\right)+1\right)=z\left(v^{3}+v+v^{2}+1\right)$ et $z(v(1+u)+u)=z v^{3}$.

\subsection{Calcul du nombre des $\alpha$ donnés par la proposition 5.2}

On peut évaluer le nombre des $\alpha$ qui donnent chaque cas de la proposition précédente.

\subsubsection{Le nombre des $\alpha$ tels que $X_{\alpha}=2 q$}

D'abord, on évalue le nombre des $\alpha$ tels que $\operatorname{Tr} \ell=1$ dans la proposition 5.2 .

Proposition 5.3 Le nombre $N_{0}$ des valeurs de $\alpha$ telles que $X_{\alpha}=2 q$ vérifie

$$
\left|N_{0}-\frac{q}{2}\right|<3 q^{1 / 2} \text {. }
$$

Démonstration -

On a $\operatorname{Tr} \ell=\operatorname{Tr}\left(a_{7}^{-1 / 3} \alpha^{-7 / 3}\right)$. Le nombre de $\alpha$ dans $k^{*}$ tels que $\operatorname{Tr}\left(a_{7}^{-1 / 3} \alpha^{-7 / 3}\right)=$ 1 est égal au nombre $N_{0}$ de $x$ dans $k^{*}$ tels que $\operatorname{Tr}\left(a_{7}^{-1 / 3} x^{7}\right)=1$. Définissons

$$
S_{0}=\sum_{x \in k} \chi\left(a_{7}^{-1 / 3} x^{7}\right)=N_{0}-\left(q-N_{0}\right)=2 N_{0}-q .
$$

On a $\left|S_{0}\right|<6 \sqrt{q}$ d'où

$$
\frac{q-6 \sqrt{q}}{2} \leq N_{0}=\frac{S_{0}+q}{2} \leq \frac{q+6 \sqrt{q}}{2} .
$$




\subsubsection{Une courbe auxiliaire}

On a besoin d'évaluer le nombre des $(\alpha, v)$ vérifiant certaine conditions, avec $v$ tel que $v+v^{4}=\ell=a_{7}^{-1 / 3} \alpha^{-7 / 3}$. Soit $x^{-3}=\alpha$ et $a_{7}^{-1 / 3}=\gamma$.

Proposition 5.4 On considère la courbe $C$ donnée par l'équation

$$
v+v^{4}=\gamma x^{7}
$$

avec les coordonnées $x$ et $v$ et le modèle non singulier $\tilde{C}$. Alors le morphisme $\tilde{C} \longrightarrow C$ est bijectif. La courbe a un unique point à l'infini. Elle est de genre 9. Les valuations au point $(0,0)$ sont $v_{(0,0)}(x)=1$ et $v_{(0,0)}(v)=7$. Les valuations au point à l'infini sont $v_{\infty}(x)=-4$ et $v_{\infty}(v)=-7$.

Démonstration -

Voir le livre de Stichtenoth [20] p. 200.

\subsubsection{Bornes pour les sommes exponentielles}

Sur la courbe $\tilde{C}$, on considère une fonction rationnelle $f$, qui n'est pas de la forme $\phi^{2}+\phi$, avec $\phi$ un fonction rationnelle sur $\tilde{C}$. Soit

$$
S=\sum_{z \in \tilde{C}_{0}(k)}{ }^{\prime} \chi(f(z))
$$

où la somme est définie sur les points rationnels sur $k$ de $\tilde{C}$, qui ne sont pas des pôles de $f$. Soit $(f)_{\infty}$ le diviseur des pôles de $f$ et $t$ le nombre de pôles de $f$, sans multiplicité. La proposition suivante donne une borne pour les sommes exponentielles $S$.

Proposition 5.5 On a

$$
|S| \leq\left(2 g-2+t+\operatorname{deg}(f)_{\infty}\right) \sqrt{q}
$$

Démonstration -

Voir l'article de Bombieri, 幽.

\subsubsection{Le nombre des $(\alpha, v)$ tels que $\operatorname{Tr}\left(\eta v^{3}\right)=1$}

On évalue le nombre des $(\alpha, v)$ tels que $\operatorname{Tr}\left(\eta v^{3}\right)=1$, où $\eta$ est donné par (4). 
On a

$$
\begin{aligned}
\operatorname{Tr}\left(\eta v^{3}\right) & =\operatorname{Tr}\left(v^{3}+\sum_{0}^{s} v^{3}\left(b_{i} \alpha^{1+2^{i}}\right)^{2^{-i}}+\sum_{0}^{s} v^{3} b_{i} \alpha^{1+2^{i}}+v^{3} \sqrt{a_{7}} \alpha^{7 / 2}+v^{3} a_{7}^{1 / 4} \alpha^{7 / 4}\right) \\
& =\operatorname{Tr}\left(v^{3}+\sum_{0}^{s}\left(v^{3.2^{i}}+v^{3}\right) b_{i} x^{-3-3.2^{i}}+\left(v^{6}+v^{12}\right) a_{7} x^{-21}\right) .
\end{aligned}
$$

Sur la courbe $C$, on considère la fonction

$$
f(x)=v^{3}+\sum_{0}^{s}\left(v^{3.2^{i}}+v^{3}\right) b_{i} x^{-3-3.2^{i}}+\left(v^{6}+v^{12}\right) a_{7} x^{-21} .
$$

Pour vérifier que $f$ n'est pas de la forme $\phi^{2}+\phi$, on considère

$$
\psi=\gamma^{1 / 4} \frac{x}{v}\left(v^{3} x^{-3}\right)^{2^{i-2}}=\gamma^{1 / 4}\left(v x^{-1}\right)^{3.2^{i-2}-1} .
$$

Si $i \geq 2$, on a

$$
\begin{aligned}
\left(v^{3.2^{i}}+v^{3}\right) x^{-3-3.2^{i}}+\psi^{4}+\psi & =\left(\frac{x^{-3}\left(\gamma x^{7}+v\right)+\gamma x^{4}}{v^{4}}\right)\left(v^{3} x^{-3}\right)^{2^{i}}+v^{3} x^{-3-3.2^{i}}+\psi \\
& =\left(x^{-3} v^{-3}\right)\left(v^{3} x^{-3}\right)^{2^{i}}+v^{3} x^{-3-3.2^{i}}+\psi
\end{aligned}
$$

Et sa valuation à l'infini est donnée par

$$
\begin{aligned}
v_{\infty}\left(\left(v^{3.2^{i}}+v^{3}\right) x^{-3-3.2^{i}}+\psi^{4}+\psi\right) & =v_{\infty}\left(\left(x^{-3} v^{-3}\right)\left(v^{3} x^{-3}\right)^{2^{i}}+v^{3} x^{-3-3.2^{i}}+\psi\right) \\
& =33-9.2^{i}
\end{aligned}
$$

si $i \geq 3$. C'est un entier négatif impair.

En faisant de même pour chaque entier $i$ dans l'expression de $f$, on trouve une fonction $\psi$ telle que la valuation au point à l'infini de $f+\psi^{2}+\psi$ soit un entier impair négatif.

On peut vérifier que la fonction $f$ est définie sur chaque point fini de $C$ sauf peut-être en les points tels que $x=0$.

On considère la somme

$$
S_{1}=\sum_{(x, v) \in C(k)-C_{\infty}} \chi(f)
$$

où $C_{\infty}=\left\{(0,0),(0,1),(0, \beta),\left(0, \beta^{2}\right), \infty\right\}$ et $\beta$ est une racine primitive $3^{\text {ème }}$ de l'unité. Les pôles de $f$ ne peuvent être que parmi les points dans $C_{\infty}$. La valuation de $f$ à l'infini est

$$
v_{\infty}(f) \geq \inf \left(v_{\infty}\left(v^{3}\right), v_{\infty}\left(b_{s} x^{-3\left(1+2^{s}\right)} v^{3.2^{s}}\right)\right) \geq-9.2^{s}+12
$$


si $s \geq 2$. La valuation de $f$ en $(0,0)$ est

$$
v_{(0,0)}(f)=v_{(0,0)}\left(v^{3} x^{-3-3.2^{s}}\right)=21-3\left(1+2^{s}\right)=18-3.2^{s} .
$$

La valuation de $v^{3.2^{i}}+v^{3}$ en $(0,1)$ est

$$
v_{(0,1)}\left(v^{3.2^{i}}+v^{3}\right)=v_{(0,1)}\left(\left(v^{3}+1\right) \prod_{\delta \in \mathbf{F}_{2^{i}}-\{1\}}\left(v^{3}-\delta\right)\right)=v_{(0,1)}\left(\frac{x^{7}}{v}\right)=7 .
$$

La valuation de $\left(v^{3.2^{i}}+v^{3}\right) x^{-3-3.2^{i}}$ en $(0,1)$ est donc

$$
v_{(0,1)}\left(v^{3.2^{i}}+v^{3}\right) x^{-3-3.2^{i}}=7-3-3.2^{i}=4-3.2^{i} .
$$

La valuation de $v^{6}+v^{12}$ en $(0,1)$ est

$$
v_{(0,1)}\left(v^{6}+v^{12}\right)=2 v_{(0,1)}\left(1+v^{3}\right)=2 v_{(0,1)}\left(x^{7}\right)=14 .
$$

La valuation de $\left(v^{6}+v^{12}\right) x^{-21}$ en $(0,1)$ est

$$
v_{(0,1)}\left(\left(v^{6}+v^{12}\right) x^{-21}\right)=14-21=-7 .
$$

La valuation de $f$ en $(0,1)$ est finalement

$$
v_{(0,1)}(f)=\inf \left(4-3.2^{s},-7\right)=4-3.2^{s}
$$

si $4-3.2^{s}<-7$ c'est-à-dire si $s \geq 2$.

Le même calcul vaut pour la valuation de $f$ en $(0, \beta)$. On a donc, pour $s \geq 2$ :

$$
\operatorname{deg}(f)_{\infty}=-3\left(4-3.2^{s}\right)-\left(18-3.2^{s}\right)-12+9.2^{s}=-42+21.2^{s}
$$

et

$$
\left|S_{1}\right| \leq\left(18-2+5-42+21.2^{s}\right) q^{1 / 2}=\left(21.2^{s}-21\right) q^{1 / 2} .
$$

Considérons sur la courbe $C$ le nombre $N_{1}$ des couples $(\alpha, v)$ tels que $\operatorname{Tr}\left(\eta v^{3}\right)=1$. Alors

$$
S_{1}=\sum_{(x, v) \in C-C_{\infty}} \chi(f)=\sum_{\operatorname{Tr} f=0} 1-N_{1}=\# C-2 N_{1}-5
$$

où $\# C$ est le nombre des points de la courbe $C$. Donc

$$
\left|N_{1}-\frac{\# C}{2}\right|=\frac{\left|S_{1}+5\right|}{2} \leq \frac{21.2^{s}-21}{2} q^{1 / 2}+5 / 2 .
$$




\subsubsection{Le nombre des $(\alpha, v)$ tels que $\operatorname{Tr}\left(\eta\left(v^{2}+v\right)\right)=1$}

Ensuite, nous évaluons le nombre des $(\alpha, v)$ tels que $\operatorname{Tr}\left(\eta\left(v^{2}+v\right)\right)=1$, où $\eta$ est donné par (4).

$$
\begin{aligned}
\operatorname{Tr}\left(\eta\left(v^{2}+v\right)\right) & =\operatorname{Tr}\left(\left(a_{7}^{1 / 4} \alpha^{7 / 4}+a_{7}^{1 / 2} \alpha^{7 / 2}+\left(\sum_{0}^{s}\left(b_{i} \alpha^{1+2^{i}}\right)^{2^{-i}}+\sum_{0}^{s} b_{i} \alpha^{1+2^{i}}\right)\right)\left(v^{2}+v\right)\right) \\
& =\operatorname{Tr}\left(a_{7} \gamma^{2} x^{-7}+\sum_{0}^{s}\left(b_{i} x^{-3\left(1+2^{i}\right)}\right)\left(v^{2^{i+1}}+v^{2^{i}}+v^{2}+v\right)\right) .
\end{aligned}
$$

On définit la fonction $g(x)=a_{7} \gamma^{2} x^{-7}+\sum_{0}^{s}\left(b_{i} x^{-3\left(1+2^{i}\right)}\right)\left(v^{2^{i+1}}+v^{2^{i}}+v^{2}+v\right)$. Elle n'est pas de la forme $\phi^{2}+\phi$ parce que avec $\psi=\gamma^{1 / 2} x^{2-3.2^{s-1}}$, on a

$$
\begin{aligned}
v_{0,0}\left(x^{-3\left(1+2^{s}\right)} v+\psi^{2}+\psi\right) & =v_{0,0}\left(x^{-3\left(1+2^{s}\right)} v+\gamma\left(x^{4-3.2^{s}}\right)+\gamma^{1 / 2} x^{2-3.2^{s-1}}\right) \\
& =v_{0,0}\left(x^{-3.2^{s}}\left(x^{-3}\left(v^{4}+\gamma x^{7}\right)+\gamma x^{4}\right)+\gamma^{1 / 2} x^{2-3.2^{s-1}}\right) \\
& \geq \inf \left(-3.2^{s}+25,2-3.2^{s-1}\right)
\end{aligned}
$$

d'où

$$
v_{0,0}\left(x^{-3\left(1+2^{s}\right)}\left(v^{2}+v\right)+\psi^{2}+\psi\right)=-3.2^{s}+11
$$

car $-3.2^{s}+11<-3.2^{s}+25$ et $-3.2^{s}+11<2-3.2^{s-1}$ si $3<2^{s-1}$ c'est-àdire si $s \geq 3$. Si $s=2$, on obtient le même résultat. En tout état de cause, $v_{0,0}\left(x^{-3\left(1+2^{s}\right)} v+\psi^{2}+\psi\right)$ est un entier impair négatif.

La valuation de $x^{-21}\left(v^{8}+v^{2}\right)=x^{-7}$ en $\infty$ est

$$
v_{\infty}\left(x^{-21}\left(v^{8}+v^{2}\right)\right)=84-7.8=28 .
$$

La valuation de $\left(b_{i} x^{-3\left(1+2^{i}\right)}\right)\left(v^{2^{i+1}}+v^{2^{i}}+v^{2}+v\right)$ en $\infty$ est

$$
v_{\infty}\left(x^{-3\left(1+2^{i}\right)}\left(v^{2^{i+1}}+v^{2^{i}}+v^{2}+v\right)\right)=12\left(1+2^{i}\right)-7.2^{i+1}=-2.2^{i}+12 .
$$

Donc la fonction $g$ a pour valuation à l'infini

$$
v_{\infty}(g)=-2.2^{i}+12 .
$$

La valuation de $x^{-21}\left(v^{8}+v^{2}\right)=x^{-7}$ en $(0,0), \ldots,\left(0, \beta^{2}\right)$ est

$$
v_{(0,0)}\left(x^{-21}\left(v^{8}+v^{2}\right)\right)=-21+7.2=-7 .
$$


La valuation de $\left.\left(b_{i} x^{-3\left(1+2^{i}\right)}\right)\left(v^{2^{i+1}}+v^{2^{i}}+v^{2}+v\right)\right)$ en $(0,0)$ est

$$
-3\left(1+2^{i}\right)+7=4-3.2^{i}
$$

La valuation de $g$ en $(0,0)$ est

$$
v_{(0,0)}(g)=4-3.2^{s}
$$

si $4-3.2^{s}<-7$, c'est-à-dire si $\frac{11}{3}<2^{s}$ c'est-à-dire si $s \geq 2$.

La valuation de $\left.\left(v^{2}+v\right)\right)$ en $(0,1)$ est

$$
v\left(v^{2}+v\right)=v\left(\frac{v^{4}+v}{1+v+v^{2}}\right)=v\left(\frac{x^{7}}{1+v+v^{2}}\right)=7 .
$$

La valuation de $\left.\left(b_{i} x^{-3\left(1+2^{i}\right)}\right)\left(v^{2^{i+1}}+v^{2^{i}}+v^{2}+v\right)\right)$ en $(0,1)$ est

$$
\left.v_{(0,0)}\left(x^{-3\left(1+2^{i}\right)}\right)\left(v^{2^{i+1}}+v^{2^{i}}+v^{2}+v\right)\right)=-3\left(1+2^{i}\right)+7=4-3.2^{i} .
$$

La valuation de $v^{2^{i+1}}+v^{2^{i}}+v^{2}+v$ en $(0, \beta)$ est

$$
\begin{aligned}
v_{(0, \beta)}\left(v^{2^{i+1}}+v^{2^{i}}+v^{2}+v\right) & =v_{(0, \beta)}\left(\left(v^{2}+v\right)^{2^{i}}+v^{2}+v\right) \\
& =v_{(0, \beta)}\left(v^{2}+v+1\right) \\
& =7 .
\end{aligned}
$$

La valuation de $\left.\left(b_{i} x^{-3\left(1+2^{i}\right)}\right)\left(v^{2^{i+1}}+v^{2^{i}}+v^{2}+v\right)\right)$ en $(0, \beta)$ est

$$
v_{(0, \beta)}\left(\left(b_{i} x^{-3\left(1+2^{i}\right)}\right)\left(v^{2^{i+1}}+v^{2^{i}}+v^{2}+v\right)\right)=-3\left(1+2^{i}\right)+7=4-3.2^{i} .
$$

Donc la valuation de $g$ en $(0,1),(0, \beta),\left(0, \beta^{2}\right)$ est

$$
v_{(0, v)}(g)=4-3.2^{s}
$$

si $4-3.2^{s}<-7$, c'est-à-dire si $\frac{11}{3}<2^{s}$ c'est-à-dire si $s \geq 2$.

Calculons maintenant

$$
S_{2}=\sum_{(x, v) \in C(k)-C_{\infty}} \chi(g) .
$$

La valuation de $g$ en chacun de ces points finis est supérieure à la plus faible des valuations de $\left(v^{2}-v^{8}\right) / x^{21}$ et $\left(b_{i} x^{-3\left(1+2^{i}\right)}\right)\left(v^{2^{i+1}}+v^{2^{i}}+v^{2}+v\right)$, elle est donc plus grande que $4-3.2^{s}$. La valuation de $g$ à l'infini est supérieure à la 
plus faible des valuations de $\left(v^{2}-v^{8}\right) / x^{21}$ et $\left(b_{i} x^{-3\left(1+2^{i}\right)}\right)\left(v^{2^{i+1}}+v^{2^{i}}+v^{2}+v\right)$, elle est donc plus grande que $12-2^{s+1}$. Donc

$$
\operatorname{deg}(g)_{\infty} \leq 4\left(-4+3.2^{s}\right)-12+2^{s+1}=14.2^{s}-28 .
$$

Par conséquent, on a

$$
\left|S_{2}\right| \leq\left(18-2+5+14.2^{s}-28\right) q^{1 / 2}=7\left(2^{s+1}-1\right) q^{1 / 2} .
$$

Soit $N_{2}$ le nombre des couples $(\alpha, v)$ tels que $\operatorname{Tr}\left(\eta\left(v^{2}+v\right)\right)=1$. Alors

$$
S_{2}=\sum_{(x, v) \in C-C_{\infty}} \chi(g)=\sum_{\operatorname{Tr} g=0} 1-N_{2}=\# C-2 N_{2}-5
$$

car $\# C_{\infty}=5$. Donc

$$
\left|N_{2}-\frac{\# C}{2}\right|=\frac{\left|S_{2}+5\right|}{2} \leq \frac{7}{2}\left(2^{s+1}-1\right) q^{1 / 2}+\frac{5}{2} .
$$

\subsubsection{Le nombre des $(\alpha, v)$ tels que $\operatorname{Tr}\left(\eta\left(v^{2}+v\right)\right)=\operatorname{Tr}\left(\eta v^{3}\right)$}

Ensuite, nous évaluons le nombre des $(\alpha, v)$ tels que $\operatorname{Tr}\left(\eta\left(v^{2}+v\right)\right)=$ $\operatorname{Tr}\left(\eta v^{3}\right)$ c'est-à-dire $\operatorname{Tr}\left(\eta\left(v^{3}+v^{2}+v\right)\right)=0$. Nous avons à calculer le nombre des $(x, v)$ tels que

$$
\operatorname{Tr}(g(x)+f(x))=0 .
$$

On considère la somme

$$
S_{3}=\sum_{(x, v) \in C(k)-C_{\infty}} \chi(f+g) .
$$

Pour vérifier que $f+g$ n'est pas de la forme $\phi^{2}+\phi$, il suffit de calculer valuation en $(0,0)$ de $f+g+b_{s} \phi^{2}+b_{s}^{1 / 2} \phi$ comme dans la sous-section précédente (5.5.5). On a

$$
v_{(0,0)} f=18-3.2^{s} \quad \text { et } \quad v_{(0,0)}\left(g+b_{s} \phi^{2}+b_{s}^{1 / 2} \phi\right)=11-3.2^{s} .
$$

On obtient dans tous les cas une valuation impaire négative.

Par l'analyse précédente, on a

$$
\operatorname{deg}(f+g)_{\infty}=21.2^{s}-63+14.2^{s}-28=35.2^{s}-91
$$


Donc on a

$$
\left|S_{3}\right| \leq\left(18-2+5+35.2^{s}-91\right) q^{1 / 2}=\left(35.2^{s}-70\right) q^{1 / 2}
$$

Soit $N_{3}$ le nombre des couples $(\alpha, v)$ tels que $\operatorname{Tr}\left(\eta\left(v^{3}+v^{2}+v\right)\right)=0$. Alors

$$
S_{3}=\sum_{(x, v) \in C-C_{\infty}} \chi(f+g)=N_{3}-\sum_{\operatorname{Tr} f+g=1} 1=2 N_{3}-\# C+5
$$

car $\# C_{\infty}=5$. Donc

$$
\left|N_{3}-\frac{\# C}{2}\right|=\frac{\left|S_{3}+5\right|}{2} \leq \frac{1}{2}\left(35.2^{s}-70\right) q^{1 / 2}+\frac{5}{2} .
$$

\subsubsection{Le nombre des $\alpha$ tels que $X_{\alpha}=8 q$}

Nous avons besoin d'un lemme.

Lemme 5.1 Soient deux fonctions $\phi$ et $\psi$ définies sur un ensemble fini $X$ à valeurs dans $\mathbf{F}_{2}$. Supposons que

$$
\begin{aligned}
\#\{x: \phi(x)=0\} & =N_{1} \\
\#\{x: \psi(x)=0\} & =N_{2} \\
\#\{x: \phi(x)=\psi(x)\} & =N_{3}
\end{aligned}
$$

Alors

$$
\#\{x: \phi(x)=\psi(x)=0\}=\frac{1}{2}\left(N_{1}+N_{2}+N_{3}-N\right)
$$

où $N$ est le nombre d'éléments de $X$.

\section{Démonstration -}

Posons

$$
\begin{array}{rll}
\{x: \phi(x)=\psi(x)=0\}=N_{0,0} & , & \{x: \phi(x)=0, \psi(x)=1\}=N_{0,1} \\
\{x: \phi(x)=1, \psi(x)=0\}=N_{1,0} & , & \{x: \phi(x)=\psi(x)=1\}=N_{1,1}
\end{array}
$$

On a

$$
N_{0,0}+N_{0,1}=N_{1} \quad, \quad N_{0,0}+N_{1,0}=N_{2} \quad, \quad N_{0,0}+N_{1,1}=N_{3}
$$

La somme des $N_{i, j}$ étant égale à $N$, on a donc

$N=\sum N_{i, j}=N_{0,0}+\left(N_{1}-N_{0,0}\right)+\left(N_{2}-N_{0,0}\right)+\left(N_{3}-N_{0,0}\right)=N_{1}+N_{2}+N_{3}-2 N_{0,0}$

D'où

$$
N_{0,0}=\frac{N_{1}+N_{2}+N_{3}-N}{2}
$$


Proposition 5.6 Le nombre $N$ des valeurs de $\alpha$ telles que $X_{\alpha}=8 q$ vérifie

$$
\left|N-\frac{q}{8}\right|<23.2^{s-1} q^{1 / 2}
$$

pour $q \geq 32$.

Démonstration -

D'après la proposition 5.2, il faut calculer le nombre $N^{\prime}$ des points $(x, v)$ tels que $\operatorname{Tr}\left(\eta v^{3}\right)=1$ et $\operatorname{Tr}\left(\eta\left(v^{2}+v\right)\right)=1$. D'après le lemme 5.1, ce nombre vérifie

$$
\begin{aligned}
N^{\prime} & =\frac{1}{2}\left(N_{1}+N_{2}+N_{3}-\# C\right) \\
& =\frac{1}{2}\left(N_{1}-\frac{\# C}{2}+N_{2}-\frac{\# C}{2}+N_{3}-\frac{\# C}{2}\right)+\frac{\# C}{4}
\end{aligned}
$$

et on a

$$
\begin{aligned}
\left|N^{\prime}-\frac{\# C}{4}\right| & =\left|\frac{1}{2}\left(N_{1}-\frac{\# C}{2}+N_{2}-\frac{\# C}{2}+N_{3}-\frac{\# C}{2}\right)\right| \\
& \leq \frac{1}{2}\left(\frac{21.2^{s}-21}{2} q^{1 / 2}+5 / 2+\frac{7}{2}\left(2^{s+1}-1\right) q^{1 / 2}+\frac{5}{2}+\frac{1}{2}\left(35.2^{s}-70\right) q^{1 / 2}+\frac{5}{2}\right) \\
& \leq\left(15 / 4-25 q^{1 / 2}+91.2^{(s-2)} q^{1 / 2}\right) .
\end{aligned}
$$

Comme pour chaque $\alpha$ tel que $\operatorname{Tr}\left(\eta v^{3}\right)=1$ et $\operatorname{Tr}\left(\eta\left(v^{2}+v\right)\right)=1$ il y a deux valeurs de $v$ (soit $v$ et $v+1$ ), le nombre $N$ de tels $\alpha$ vérifie donc

$$
\left|N-\frac{\# C}{8}\right| \leq\left(15 / 8-25 / 2 . q^{1 / 2}+91.2^{(s-3)} q^{1 / 2}\right)
$$

Comme $m$ est impair, il existe une solution de $v+v^{4}=\gamma x^{7}$ si et seulement si la trace $\operatorname{Tr}\left(\gamma x^{7}\right)$ est nulle et, dans ce cas, il y a exactement deux solutions. Donc

$$
\# C(k)=2 \#\left\{\operatorname{Tr}\left(\gamma x^{7}\right)=0\right\}+1=S_{7}+q+1
$$

où $S_{7}$ est la somme exponentielle $S_{7}=\sum_{x \in k}(-1)^{\operatorname{Tr}\left(\gamma x^{7}\right)}$. Donc

$$
|\# C(k)-q-1| \leq 6 \sqrt{q} .
$$

On a

$$
\left|N-\frac{q}{8}\right| \leq\left|N-\frac{\# C}{8}\right|+\left|\frac{\# C}{8}-\frac{q}{8}-\frac{1}{8}\right|+\frac{1}{8} .
$$

Donc le nombre $N$ vérifie

$$
\left|N-\frac{q}{8}\right| \leq 15 / 8-25 / 2 . q^{1 / 2}+91.2^{(s-3)} q^{1 / 2}+\frac{3}{4} q^{1 / 2}+\frac{1}{8} \leq 23.2^{s-1} q^{1 / 2} .
$$




\section{Démonstration de l'évaluation de $\|\widehat{f}\|_{4}^{4}$ (propo- sition 4.1)}

On déduit facilement de la proposition 5.6 le calcul de la valeur de $\|\widehat{f}\|_{4}^{4}$. Sachant que

$$
\begin{aligned}
& \left|N-\frac{q}{8}\right| \leq 23.2^{s-1} q^{1 / 2} \\
& \left|N_{0}-\frac{q}{2}\right| \leq 3 q^{1 / 2}+1
\end{aligned}
$$

calculons

$$
\begin{aligned}
\|\widehat{f}\|_{4}^{4} & =q^{2}+\sum_{\substack{\alpha \neq 0 \\
\alpha \in V_{m}}} X_{\alpha} \\
& =3 q^{2}+8 q(N-q / 8)+2 q\left(N_{0}-q / 2\right) .
\end{aligned}
$$

D'où

$$
\begin{aligned}
\left|\|\widehat{f}\|_{4}^{4}-3 q^{2}\right| & \leq 8 q\left|N-\frac{q}{8}\right|+2 q\left|N_{0}-\frac{q}{2}\right| \\
& \leq 185.2^{s-1} q^{3 / 2} .
\end{aligned}
$$

\section{Démonstration des bornes de $\|\widehat{f}\|_{\infty}$ (propo- sitions 4.2 et 4.3 )}

\subsection{Borne inférieure}

L'évaluation du nombre des $\alpha$ tels que $\operatorname{Tr} \ell=1$ dans la proposition 5.2 donne :

$$
2 q^{2}-6 q^{3 / 2} \leq\|\widehat{f}\|_{4}^{4}
$$

On a

$$
\sum_{\alpha \in k^{*}} X_{\alpha} \geq 2 q N \geq 2 q \frac{q-6 \sqrt{q}}{2}=q^{2}-6 q^{3 / 2}
$$

et

$$
\|\widehat{f}\|_{4}^{4}=q^{2}+\sum_{\alpha \in k^{*}} X_{\alpha} \geq 2 q^{2}-6 q^{3 / 2}
$$


Comme il est facile de montrer que

$$
\|\widehat{f}\|_{4}^{4} \leq q\|\widehat{f}\|_{\infty}^{2}
$$

nous obtenons $2 q-6 q^{1 / 2} \leq\|\widehat{f}\|_{\infty}^{2}$, donc $\sqrt{2 q}-3 \sqrt{2} \leq\|\widehat{f}\|_{\infty}$, d'où le résultat si $m \geq 7$, parce que $\|\widehat{f}\|_{\infty}$ est un entier divisible par $2^{\lceil m / 3\rceil}$. Le résultat pour $m \leq 7$ est connu (cf. remarque 4.2).

On a, de plus

$$
\|\widehat{f}\|_{4}^{4} \geq 3 q^{2}-185.2^{s-1} q^{3 / 2}
$$

par la proposition 4.1. On en déduit que pour que $\|\widehat{f}\|_{4}^{4}$ dépasse $2 q^{2}$, il suffit que $m \geq 15+2 s$. Pour des raisons de divisibilité, $\|\widehat{f}\|_{\infty}$ est alors plus grand que $\sqrt{2 q}+2^{\left\lceil\frac{m}{3}\right\rceil}$.

\subsection{Borne supérieure}

On a, d'après la borne de Weil

$$
|\widehat{f}(v)|=\left|\sum_{x \in V_{m}} \chi(f(x)+v \cdot x)\right| \leq 6 \sqrt{q} .
$$

\section{Références}

[1] Anne Canteaut Differential cryptanalysis of Feistel ciphers and differentially $\delta$-uniform mappings, in Selected Areas on Cryptography, SAC'97, pages 172-184, Ottawa, Canada, 1997.

[2] A. Canteaut, C. Carlet, P. Charpin, C. Fontaine Propagation characteristics et correlation-immunity of highly nonlinear Boolean functions, Advances in cryptology, EUROCRYPT 2000 (Bruges), 507-522, Lecture Notes in Comput. Sci., Vol. 1807, Springer, Berlin, 2000.

[3] Barth, Rolland, Véron Cryptographie, Hermès, Paris, 2005.

[4] E. Bombieri, On exponential sums in finite fields. Amer. J. Math., 88, 1966, pp. 71-105.

[5] C. Carlet, On cryptographic complexity of Boolean functions, Proceedings of the Sixth Conference on Finite Fields with Applications to Coding Theory, Cryptography et Related Areas (G.L. Mullen, H. Stichtenoth et H. Tapia-Recillas Eds), Springer (2002) pp. 53-69. 
[6] C. Carlet, On the algebraic thickness et non-normality of Boolean functions, with developments on symmetric functions, submitted to IEEE Trans. Inform. Theory.

[7] G. Cohen, I. Honkala, S. Litsyn, A. Lobstein, Covering codes. NorthHolland Mathematical Library, 54, North-Holland Publishing Co., Amsterdam (1997).

[8] C. Fontaine, Contribution à la recherche de fonctions booléennes hautement non linéaires et au marquage d'images en vue de la protection des droits d'auteur, Thèse, Université Paris VI (1998).

[9] X. Hou, Covering radius of the Reed-Muller code $R(1,7)$ - a simpler proof. J. Combin. Theory Ser. A 74 (1996), no. 2, 337-341.

[10] Leander, Nils Gregor Monomial bent functions. IEEE Trans. Inform. Theory 52 (2006), no. 2, 738-743.

[11] Langevin, P. ; Zanotti, J.-P. Nonlinearity of some invariant Boolean functions. Des. Codes Cryptogr. 36 (2005), no. 2, 131-146.

[12] Selçuk Kavut, Subhamoy Maitra and Melek D. Yücel There exist Boolean functions on $n$ (odd) variables having nonlinearity $>2^{n-1}-2^{\frac{n-1}{2}}$ if and only if $n>7$, prépublication, http ://eprint.iacr.org/2006/181

[13] C. Moreno et O. Moreno The MacWilliams-Sloane conjecture on the tightness of the Carlitz-Uchiyama bound and the weights of duals of BCH codes. IEEE Trans. Inform. Theory 40 (1994), no. 6, 1894-1907.

[14] Daniel Maisner et Enric Nart, Zeta functions of supersingular curves of genus 2, arXiv :math.NT/0408383

[15] F.J. MacWilliams et N.J.A. Sloane, The Theory of Error-Correcting Codes, North-Holland, Amsterdam (1977).

[16] N. Patterson et D. Wiedemann, The covering radius of the $\left(2^{15}, 16\right)$ Reed-Muller code is at least 16276 , IEEE Trans. Inform. Theory 29, no. 3 (1983), 354-356.

[17] F. Rodier, Sur la non-linéarité des fonctions booléennes, Acta Arithmetica, vol 115, (2004), 1-22, preprint : arXiv : math.NT/0306395.

[18] F. Rodier, On the nonlinearity of Boolean functions, Proceedings of WCC2003, Workshop on coding et cryptography 2003 (D. Augot, P. Charpin, G. Kabatianski eds), INRIA (2003), pp. 397-405. 
[19] J-P. Serre, Majorations de sommes exponentielles. Journées Arithmétiques de Caen (Univ. Caen, Caen, 1976), pp. 111-126. Astérisque No. 41-42, Soc. Math.France, Paris, 1977.

[20] H. Stichtenoth, Algebraic Function Fields et Codes, Springer, 1993.

[21] P. Stănică, Nonlinearity, local et global avalanche characteristics of balanced Boolean functions, Discrete Math. 248 (2002), no. 1-3, 181-193.

[22] G. van der Geer, M. van der Vlugt, Reed-Muller codes and supersingular curves. I, Compositio Math. 84, (1992), 333-367.

[23] G. van der Geer, M. van der Vlugt, Supersingular Curves of Genus 2 over finite fields of Characteristic 2, Math. Nachr. 159, (1992), 73-81.

[24] Xian-Mo Zhang and Yuliang Zheng, GAC - the Criterion for Global Avalanche Characteristics of Cryptographic Functions, Journal of Universal Computer Science, vol. 1, no. 5 (1995), 316-333 